\title{
Evolução do conhecimento biocronoestratigráfico do Cretáceo nas bacias marginais brasileiras baseado em nanofósseis calcários
}

\author{
Rodrigo do Monte Guerra \\ Lab. de Micropaleontologia, UNISINOS. Av. Unisinos, \\ Bairro Cristo Rei, São Leopoldo. \\ rmguerra@unisinos.br \\ Lucio Riogi Tokutake \\ Dept. de Exploração, Setor de Sedimentologia e \\ Estratigrafia, Petrobras, UN-ES, Av. Fernando Ferrari, \\ 1000 Vitória. \\ tokutake@petrobras.com.br
}

\begin{abstract}
BIOCHRONOSTRATIGRAPHIC KNOWLEDGE EVOLUTION IN THE BRAZILIAN CRETACEOUS MARGINAL BASINS BASED ON CAKCAREOUS NANNOFOSSILS The use of the biostratigraphy effective contributes to the resolution of stratigraphic problems, allowing the strata correlation based on the use of fossils. Its use as a standard tool in the oil exploration, provided advances in the biostratigraphic concepts and the geological knowledge. Due to the difficulty in recover macrofossils in drilled samples, microfossils are the best alternative for correlations, paleoecological studies and chronostratigraphic placement of wells. Calcareous nannofossils is one of the most important groups, because of their abundance in rocks, the wide geographic distribution, rapid speciation and the laboratory procedure. This study aims to present the importance of the biostratigraphy and the evolution of the biochronostratigraphic knowledge of the Upper Cretaceous in the Brazilian marginal basins, by means of the main biostratigraphic zonations based on calcareous nannofossils. Citation: Guerra R. do M., Tokutake L. R. 2011. Evolução do conhecimento biocronoestratigráfico do Cretáceo nas bacias marginais brasileiras baseado em nanofósseis calcários. Terræ Didatica, 7(1):41-48<http://www.ige.unicamp.br/terraedidatica/>
\end{abstract}

\section{KEYWORDS Microfossils, calcareous nannofossils, biostratigraphy, Cretaceous.}

RESUMO O advento da bioestratigrafia contribuiu em grande parte para a resolução de problemas estratigráficos, possibilitando a correlação dos estratos rochosos com base na análise dos fósseis. A utilização como ferramenta padrão na indústria do petróleo propiciou um grande avanço nos conceitos da bioestratigrafia e no conhecimento geológico. Devido a dificuldade de recuperação de macrofósseis na amostragem em perfurações petrolíferas, os microfósseis transformaram-se em ótima alternativa para correlações, estudos paleoecológicos e posicionamento cronoestratigráfico de poços. Dentre os grupos mais utilizados estão os nanofósseis calcários, devido à abundância com que ocorrem nas rochas aliada à ampla distribuição geográfica, rápida especiação e ao fato de serem facilmente recuperados da matriz sedimentar e preparados em laboratório. Este trabalho busca apresentar a importância da bioestratigrafia e a evolução do conhecimento cronoestratigráfico do Cretáceo Superior nas bacias marginais brasileiras, por meio dos principais biozoneamentos propostos com nanofósseis calcários.

PALAVRAS-CHAVE Microfósseis, nanofósseis calcários, bioestratigrafia, Cretáceo.

\section{Introdução}

O estudo dos nanofósseis calcários somado aos conceitos da bioestratigrafia, propiciou a criação de uma ferramenta indispensável para ampliar o conhecimento sobre a idade dos estratos depositados nas bacias sedimentares marginais brasileiras.

O primeiro registro de nanofósseis calcários foi relatado por Ehrenberg (1836) (apud Sies- ser, 1994), em amostras cretácicas e eocênicas da ilha Rügen no mar Báltico, Alemanha. O autor descreve objetos elípticos, discóides e achatados apresentando um ou poucos anéis concêntricos em sua superfície, classificando-os como de origem inorgânica, produto de concreções formadas por crescimento radial de cristais de carbonato de 
cálcio em torno de um núcleo. Após o estudo de Ehrenberg, diversas interpretações sobre a origem dos nanofósseis foram publicadas. Alguns autores relacionaram os nanofósseis calcários a foraminíferos (Sorby 1861, Wallich 1861). Somente no final do século XIX, foi proposta a primeira classificação moderna segundo a qual os nanofósseis calcários correspondem ao esqueleto de diminutas algas unicelulares calcárias (Tizard et al. 1885, Murray \& Renard, 1891).

A utilidade dos nanofósseis calcários em estudos de bioestratigrafia foi divulgada por Bramlette \& Riedel (1954), porém somente no final da década de 1960, com o advento de projetos de perfuração oceânica como o Deep Sea Drilling Project (DSDP), pode-se perceber a importância estratigráfica deste grupo.

\section{Nanofósseis calcários}

De acordo com Bown \& Young (1998) o termo nanofósseis calcários refere-se à elementos predominantemente planctônicos, de composição carbonática, com dimensões inferiores a $30 \mu \mathrm{m}$ pertencentes a duas grandes categorias: os cocólitos e as formas associadas (Figura 1).

Os cocólitos, geralmente circulares a elipsoidais, são provenientes da desagregação do esqueleto externo de algas unicelulares conhecidas como cocolitoforídeos. Já as formas associadas, de conformação mais variada, referem-se a partículas orgânicas taxonomicamente indefinidas (incertae sedis) ou provenientes de outros organismos não relacionados aos cocolitoforídeos, como dinoflagelados calcários (Antunes 1997).
Os nanofósseis calcários surgiram no Triássico Superior e são encontrados em grande quantidade em vasas e sedimentos marinhos de granulometria fina, como folhelhos, siltitos argilosos e margas. No Brasil, ocorrem nas seções marinhas de todas as bacias da margem continental, da Bacia de Pelotas até a da Foz do Amazonas.

\section{Bioestratigrafia}

O estudo da bioestratigrafia objetiva a divisão de uma seção rochosa em unidades bioestratigráficas, baseado no seu conteúdo fossilífero (Antunes \& Melo 2001). Os fundamentos da bioestratigrafia foram criados pelo inglês William Smith, que em 1815 utilizou pela primeira vez o conteúdo fossilífero como ferramenta para mapear as rochas (Winchester 2004), enfatizando a sua posição estratigráfica. Smith foi o primeiro a reconhecer a importância dos fósseis no estudo de rochas sedimentares, que podem ser caracterizadas e correlacionadas com base no seu conteúdo fossilífero. A aplicação da bioestratigrafia teve início com a análise de macrofósseis, entretanto com o aprimoramento dos estudos micropaleontológicos, esta ferramenta adquiriu uma grande importância para estudos cronoestratigráficos e paleoecológicos.

A unidade fundamental na bioestratigrafia é a biozona, pacote rochoso caracterizado pelo seu conteúdo fossilífero, que permite diferenciá-lo dos estratos sobre e subjacentes. Para caracterizar uma biozona é importante que o táxon possua uma ampla distribuição geográfica, curta distribuição temporal e alto potencial de preservação sendo assim abundante e de fácil identificação. Os principais

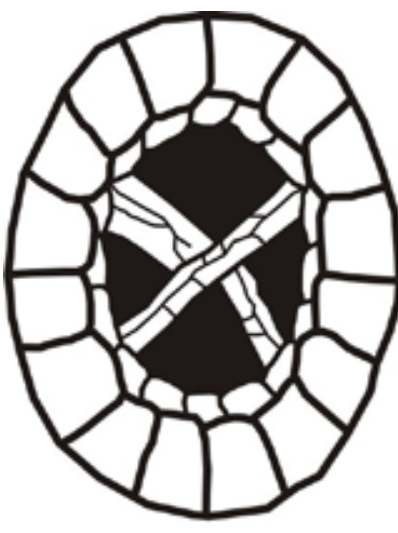

a

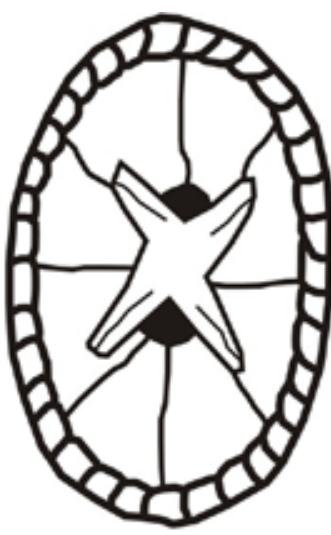

b

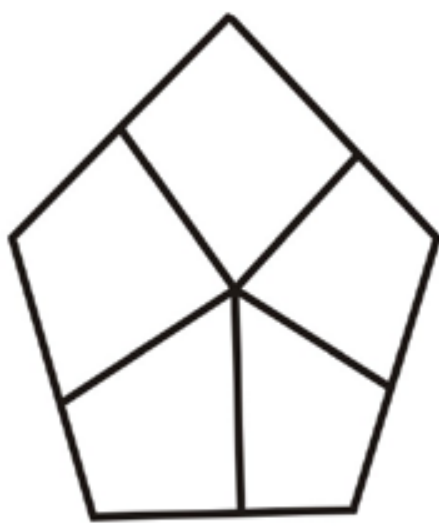

C

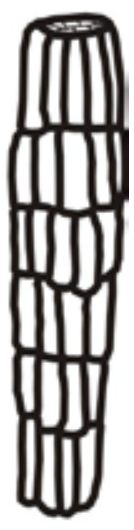

d

Figura 1. Quatro gêneros distintos exemplificando os dois grandes grupos de nanofósseis calcários, os cocólitos (a e b) e as formas associadas (c e d). a) Prediscosphaera, b) Eiffellithus, c) Braarudosphaera e d) Microrhabdulus. Modificado de Perch-Nielsen (1985) 


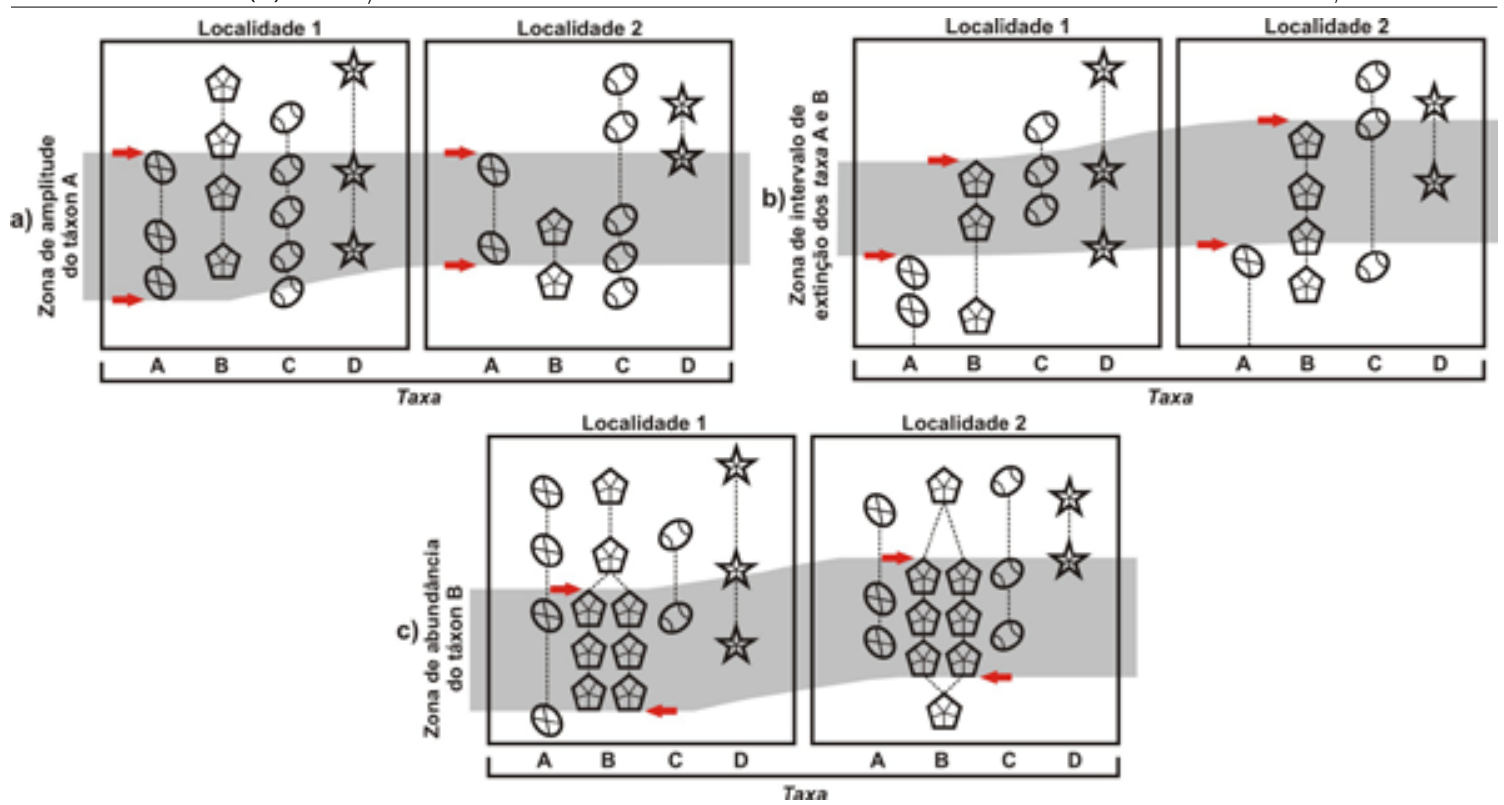

Figura 2. Principais tipos de biozona. a) Zona de amplitude indicada pela primeira e última ocorrência do táxon $A$; b) Zona de intervalo indicada pela extinção dos taxa A e B; e c) Zona de abundância do táxon B. Modificado de Zerfass \& Andrade (2008)

tipos de biozona são definidos por combinação de primeira ocorrência e extinção global de um determinado táxon (zona de amplitude), primeira ocorrência ou extinção de dois taxa distintos (zona de intervalo) e abundância de um determinado táxon (zona de abundância) (Figura 2).

$\mathrm{Na}$ indústria do petróleo a zona de intervalo com a extinção de dois taxa distintos (Figura 2b) é a mais utilizada, isso porque as amostras de calha geralmente mostram-se muito contaminadas. Essa contaminação nada mais é do que o registro concomitante de fósseis mais novos (elementos contaminantes) desabados em meio a associações de fósseis mais antigos (supostamente in situ) sendo assim, perde-se o controle do nível de surgimento das entidades taxonômicas, o que inviabiliza o reconhecimento de unidades baseadas em primeiras ocorrências (Antunes \& Melo 2001).

\section{Nanofósseis calcários e a bioestratigrafia}

Os nanofósseis calcários são empregados em estudos bioestratigráficos de depósitos marinhos, devido à abundância com que ocorrem nas rochas aliada à ampla distribuição geográfica, rápida especiação e ao fato de serem facilmente recuperados da matriz sedimentar e preparados em laboratório, o que agiliza a interpretação das análises tornando-os excelentes ferramentas para a bioestratigrafia (Antunes 1997).
A busca por uma bioestratigrafia de alta resolução utilizando nanofósseis calcários teve início entre as décadas de 1960 e 1970, após o desenvolvimento de projetos internacionais como o Deep Sea Drilling Project (DSDP) e Ocean Drilling Program (ODP), além do investimento de empresas petrolíferas.

O primeiro biozoneamento utilizando nanofósseis calcários para o período Cretáceo foi criado por Stradner (1963), com amostras de países do leste europeu. $\mathrm{O}$ autor reconheceu sete associações de espécies. Após o trabalho de Stradner, diversos autores propuseram novos biozoneamentos destacando-se Cepek \& Hay $(1969,1970)$ com amostras do Kansas e Alabama, Manitiv (1971) com amostras da França, e Roth (1973) com amostras do Pacífico central.

\subsection{Em busca de um biozoneamento internacional}

A idéia de criar um arcabouço bioestratigráfico que pudesse ser utilizado globalmente surgiu no início da década de 1970, com a análise de importantes seções cretáceas distribuídas principalmente nos continentes europeu, africano e americano. As amostras analisadas pertencem, em sua maioria, ao Deep Sea Drilling Project (DSDP); grande parte dos trabalhos citados a seguir só foi possível devido às amostragens recuperadas pelo projeto.

Thierstein (1976) produziu a primeira tentativa de um arcabouço bioestratigráfico visando aplicabi- 
lidade global. O autor utilizou amostras do DSDP, mas o biozoneamento não atingiu grande resolução, sendo imediatamente substituído.

Sissingh (1977) estabeleceu o primeiro arcabouço bioestratigráfico internacional utilizando diversas seções e alguns estratótipos principalmente continentais do oeste europeu (França, Alemanha, Inglaterra e Holanda), Tunísia, Turquia e Omã. Foram propostas 26 zonas de intervalo codificadas de CC1 (Berriasiano) a CC26 (Maastrichtiano).

Roth (1978) apresentou um novo biozoneamento com amostras de poços do DSDP próximos a costa da Flórida, no Oceano Atlântico norte. $\mathrm{O}$ autor utilizou bioeventos semelhantes aos de Sissingh (1977), provando assim a correlação bioestratigráfica de nanofósseis em sequências continentais e oceânicas. Foram propostas 23 zonas de intervalo codificadas de NC1 (Berriasiano) a NC23 (Maastrichtiano).

Perch-Nielsen (1985) reeditou o arcabouço de Sissingh (1977) apresentando um detalhamento relativamente elevado principalmente para o Campaniano e Maastrichtiano.

Burnett (1998) sugeriu um novo biozoneamen- to para o Cretáceo, justificando que os bioeventos utilizados para definir as zonas CC de Sissingh (1977) e Perch-Nielsen (1985) são inapropriados para um contexto global. Foram utilizadas amostras principalmente do DSDP e ODP de diversas partes do globo, particularmente dos oceanos Índico e Atlântico, países europeus (Dinamarca, Alemanha, Inglaterra, Polônia, França, Holanda e Espanha), Estados Unidos e África do Sul. Foram propostas 48 zonas de intervalo codificadas em NJ18 (Jurássico Superior/Cretáceo Inferior), BC1 a BC27 (Cretáceo Iinferior) e UC1 a UC20 (Cretáceo Superior).

Apesar de todo o refinamento obtido nos últimos anos, o trabalho de Sissingh (1977) ainda é o mais utilizado para correlações globais, sendo o escolhido neste estudo.

\subsection{Biozoneamento nas bacias marginais brasileiras}

As pesquisas com nanofósseis calcários nas bacias marginais brasileiras tiveram início no final da década de 1960 devido aos avanços na exploração

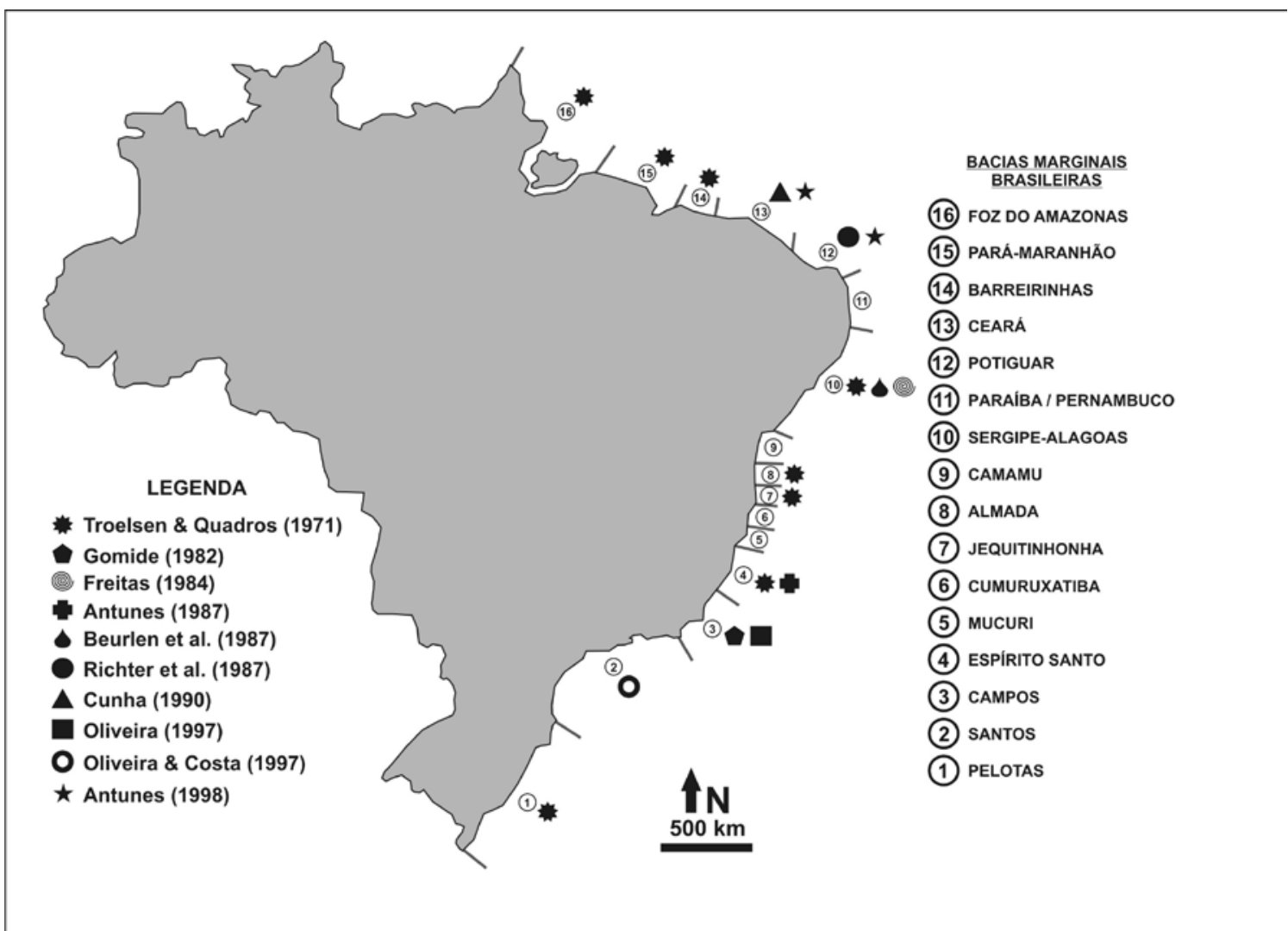

Figura 3. Mapa com a localização das bacias sedimentares marginais brasileiras. Os símbolos da legenda indicam as bacias sedimentares onde foram realizados estudos de bioestratigrafia dos nanofósseis calcários do Cretáceo Superior 
petrolífera. Os trabalhos de bioestratigrafia mais importantes para a elaboração do biozoneamento foram gerados com amostras de praticamente todas as bacias, conforme a Figura 3.

Troelsen \& Quadros (1971), publicaram o primeiro arcabouço bioestratigráfico baseado em nanofósseis calcários na margem continental brasileira, atingindo a seção Aptiano-Mioceno. Estes autores descreveram amostras de 36 poços (23 em plataforma continental e 13 no continente) localizados nos estados do Alagoas, Amapá, Bahia, Espírito Santo, Maranhão, Pará, Rio Grande do Sul e Sergipe. Para o período Cretáceo, foram individualizadas cinco zonas de amplitude: Nannoconus truitti (Aptiano superior/Albiano inferior), Lithastrinus grillii (Turoniano/Santoniano), Broinsonia parca posteriormente chamada de Aspidolithus parcus (Campaniano), Tetralithus nitidus trifidus posteriormente chamada de Uniplanarius trifidus (Campaniano/Maastrichtiano) e Arkhangelskiella cymbiformis (Maastrichtiano).

Entre as décadas de 1980 e 1990, diversos estudos foram realizados em praticamente todas as bacias marginais brasileiras, tendo como resultado o aprimoramento do arcabouço estratigráfico proposto por Troelsen \& Quadros (1971). Os trabalhos mais importantes para este refinamento são apresentados a seguir.

Gomide (1982) emendou a descrição da Zona Lithastrinus grillii de Troelsen \& Quadros (1971), com base em estudo de 76 poços na Bacia de Campos,

Freitas (1984) descreveu mais de 60 poços na Bacia de Sergipe-Alagoas, sugerindo a formalização da Zona Eiffellithus eximius (Campaniano) de Bukry \& Bramlette (1970) para a margem continental brasileira.

Antunes (1987), em estudo de 40 poços na Bacia do Espírito Santo, emendou a descrição da Zona Broinsonia parca de Troelsen \& Quadros (1971). No mesmo ano Beurlen et al. (1987) (apud Antunes, 1998), baseados em nanofósseis calcários, palinomorfos e ostracodes da Bacia de Sergipe-Alagoas, propuseram a Zona Nannoconus quadriangulus apertus (Aptiano superior/Albiano inferior).

Richter et al. (1987) analisaram um poço na Bacia Potiguar, propondo a formalização das zonas Axopodorhabdus albianus (Cenomaniano/Turoniano) e Corollithion achylosum posteriormente chamada de Stoverius achylosus (Turoniano).

Cunha (1990), em estudo de 20 poços na Bacia do Ceará, propôs a inclusão das zonas Nannoconus fragilis (Albiano), Braarudosphaera africana (Albiano/ Cenomaniano), além de formalizar a Zona Marthasterites furcatus (Coniaciano/Santoniano) de Cepek \& Hay (1969) para a margem continental brasileira e emendar a descrição da Zona Axopodorhabdus albianus de Richter et al. (1987).

Antunes (1996) apresentou um estudo com todas as biozonas propostas até o momento, com seus aspectos positivos e negativos. Em função desses aspectos idealizou um arcabouço bioestratigráfico hipotético para a margem continental brasileira. As modificações propostas pelo autor foram as seguintes: a exclusão das Zonas Nannoconus quadriangulus apertus de Beurlen et al. (1987) (apud Antunes, 1998) e Stoverius achylosus de Richter et al. (1987); emenda da descrição das Zonas Nannoconus fragilis e Braarudosphaera africana, que passou a ser denominada Zona Braarudosphaera ex. gr. B. africana, ambas de Cunha (1990); e inclusão da Zona Lithastrinus spp. (Albiano/Campaniano), dividida em cinco subzonas: Axopodorhabdus albianus (Albiano superior/Cenomaniano inferior), Radiolithus planus (Cenomaniano superior/Turoniano inferior), Eprolithus floralis (Coniaciano superior/ Santoniano inferior), Lithastrinus moratus (Santoniano) e Lithastrinus grillii (emenda da descrição de Troelsen \& Quadros (1971)).

Oliveira (1997) reconheceu as biozonas de Antunes (1996) na Bacia de Campos e incluiu na Zona Lithastrinus spp. a subzona Eprolithus eptapelatus (Turoniano inferior/Coniaciano inferior), além de emendar a descrição da subzona Eprolithus floralis de Antunes (1996).

Oliveira \& Costa (1997) propuseram um detalhamento da seção maastrichtiana com base em estudo na Bacia de Santos. Duas subzonas foram propostas para dividir a Zona Arkhangelskiella cymbiformis de Troelsen \& Quadros (1971), são as subzonas Reinhardtites levis (Campaniano/Maastrichtiano) e Arkhangelskiella spp. (Maastrichtiano).

Antunes (1998) em estudo nas bacias do Ceará e Potiguar testou o arcabouço bioestratigráfico hipotético criado por Antunes (1996) e emendado por Oliveira (1997) e Oliveira \& Costa (1997). Neste estudo, o mais completo arcabouço bioestratigráfico com nanofósseis calcários das bacias marginais brasileiras disponível no meio acadêmico, o autor refina a zona Arkhangelskiella spp. de Oliveira \& Costa (1997), dividindo-a em três subzonas: Glaukolithus compactus, Gartnerago obliquum e Cribros- 


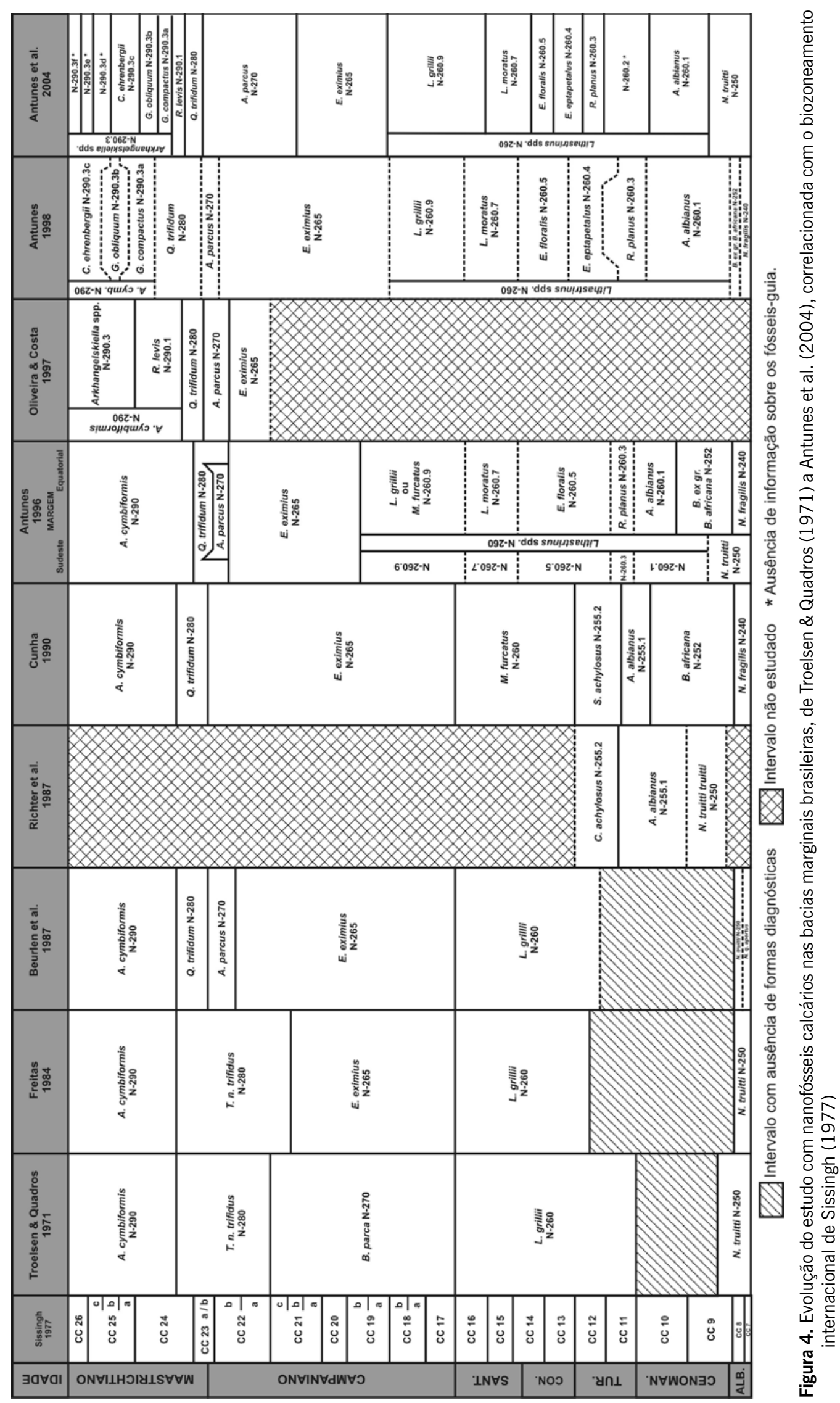


phaerella ehrenbergii (todas do Maastrichtiano).

Antunes et al. (2004) discutiram as biozonas com nanofósseis calcários do Cretáceo superior indicando a existência de mais quatro biozonas. Por se tratar de um biozoneamento padrão utilizado pela PETROBRAS, as biozonas aparecem somente pelos códigos, sem o nome dos fósseisguia (Figura 4).

\section{Discussões}

Conforme o histórico de estudos bioestratigráficos nas bacias marginais brasileiras, citados anteriormente, pôde-se perceber como os conceitos básicos da bioestratigrafia auxiliaram na evolução do arcabouço bioestratigráfico para a margem continental brasileira. O desenvolvimento de novos estudos aliados a uma melhor compreensão da distribuição estratigráfica dos fósseis-guia, conduziram a um aprimoramento do biozoneamento inicial. Uma comparação entre o biozoneamento proposto por Troelsen \& Quadros (1971) e o de Antunes et al. (2004) mostra um refinamento para o período Maastrichtiano e para a seção TuronianoSantoniano.

O aprimoramento de estudos com nanofósseis calcários nas bacias marginais brasileiras, pode auxiliar num refinamento bioestratigráfico para o Albiano, Cenomaniano e Campaniano, idades que ainda possuem uma pequena resolução estratigráfica.

\section{Agradecimentos}

Os autores agradecem aos doutores Gerson Fauth, Rogério Loureiro Antunes e Andrea Concheyro, pelo auxílio e leitura crítica do manuscrito; aos doutores Luiz Carlos Veiga de Oliveira e Ismar de Souza Carvalho, pela revisão do manuscrito, bem como aos colegas do Laboratório de Micropaleontologia da UNISINOS.

\section{Referências}

Antunes R.L. 1987. Bioestratigrafia dos nanofósseis calcários Meso e Neocretáceo da porção emersa da Bacia do Espírito Santo. B. Geoc. Petrobras, 1(1):2-11.

Antunes R.L. 1996. Biozonas de nanofósseis do Cretáceo da margem continental brasileira: problemas e possíveis soluções. B. Geoc. Peatrobrs, 10(1/4):1942.
Antunes R.L. 1997. Introdução ao estudo dos nanofósseis calcários. Rio de Janeiro: Inst. Geoc. UFRJ. 115 p.

Antunes R.L. 1998. Nanofósseis calcários e sua bioestratigrafia no Cretáceo da Margem Continental Brasileira. Bacias do Ceará e Potiguar. Rio de Janeiro: Progr. Pós-Grad.Geoc. UFRJ. 193 p. (Tese Dout.).

Antunes R.L., Melo J.H.G. 2001. Micropaleontologia e estratigrafia de seqüências. In: Ribeiro H.J.P.S. ed. Estratigrafia de Seqüências - fundamentos e aplicações. São Leopoldo: Editora Unisinos. p. 137-218.

Antunes R.L., Shimabukuro S., Oliveira L.C.V., Rosa A.L.Z., Costa S.O., Cunha A.A.S., Lima F.H.O. 2004. Em busca da bioestratigrafia de alta resolução - a performance do zoneamento de nanofósseis calcários da Petrobras. B. Geoc. Petrobras, 12(2):421-427.

Beurlen G., Freitas L.C.S, Uesugui N. Koutsoukos E.A.M. 1987. Paleoecologia do baixo de São Francisco I - Área de Brejo Grande-Carapitanga. Rio de Janeiro: Petrobrás/Cenpes/Divex/Sebipe. (Relat. Interno, Cenpes 789).

Bown P.R., Young J.R. 1998. Introduction. In: Bown P.R. ed. 1998. Calcareous Nannofossil Biostratigraphy. London: British Microp. Soc. Series. Chapman and Hall/Kluwer Acad. Publ. p. 1-15.

Bramlette M.N, Riel W.R. 1954. Stratigraphic value of discoasters and some other microfossils related to Recent coccolithophores. Journal of Paleontology, 28:385-403.

Bukry D., Bramlette M.N. 1970. Coccolith age determinations LEG 3, Deep Sea Drilling Project. Initial Reports of the DSDP, 3:589-611.

Burnett J.A. 1998. Upper Cretaceous. In: Bown P.R. ed. 1998. Calcareous Nannofossil Biostratigraphy. London: British Microp. Soc. Series. Chapman and Hall/ Klüwer Acad. Publ. p. 132-199.

Cepek P., Hay W.W. 1969. Calcareous nannoplankton and biostratigraphic subdivision of Upper Cretaceous. Trans. Gulf Coast Assoc. Geol. Soc., 19(1):323-336.

Cepek P., Hay W.W. 1970. Zonation of the Upper Cretaceous using calcareous nannoplankton. Paläobotanik, 3:333-340.

Cunha A.A.S. 1990. Bioestratigrafia de nanofósseis calcários da sub-bacia Mundaú (Bacia do Ceará). Rio Grande do Sul: Progr. Pós-Grad. Geoc. UFRGS. 151 p. (Dissert. Mestrado).

Ehrenberg C.G. 1836. Bemerkungen uber feste mikroscopische anorganische Formen in den erdigen und derben Mineralien. Berichte der Königlichen Preussischen Akademie der Wissenschaften zu Berlin, 1836: 84-85.

Freitas L.C.S. 1984. Nanofósseis calcários e sua distribuição (Aptiano-Mioceno) na Bacia Sergipe-Alagoas. Rio de Janeiro: Progr. Pós-Grad. Geoc. UFRJ, 247 p. (Dissert. Mestrado). 
Gomide J. 1982. Bioestratigrafia do nanoplâncton calcário da Bacia de Campos. B. Técn. Petrobras, 25(3):129-137.

Manivit H. 1971. Nannofossiles calcaires du Crétacé francais (Aptien-Maestrichtien). Essai de Biozonation appuyée sur les stratotypes. Faculté des Sciences d'Orsay. 187 p. (Thése Doctorate d'Etat).

Murray J., Renard A.F. 1891. Report on deep-sea deposits based on the specimens collected during the voyage of HMS Challenger in the years 1872 to 1876. In: Report on the Scientific Results of the Voyage of HMS Challenger during the years 1873-1876. London, 3:1-525.

Oliveira L.C.V. 1997. Arcabouço estratigráfico Albo-Maastrichtiano da Bacia de Campos: um estudo com base em nanofósseis calcários e suas correlações com marcos elétricos e a estratigrafia química. Rio Grande do Sul: Progr. Pós-Grad. Geoc., UFRGS. 180 p. (Dissert. Mestrado).

Oliveira L.C.V., Costa S.O. 1997. Proposal of new biostratigraphic units based on calcareous nannofossils for the Maastrichtian of the Santos Basin (Brazil). An. Acad. brasil. Ciênc., 69(1):37-58.

Perch-Nielsen K. 1985. Mesozoic calcareous nannofossils. In: Bolli H.M., Saunders J.B., Perch-Nielsen K. eds. 1985. Plankton Stratigraphy. Cambridge: Cambridge Univ. Press. p. 329-426.

Richter A.J., Freitas L.C.S., Wanderley M.D. 1987. Nanofósseis calcários: duas novas biozonas do Cretáceo médio da plataforma continental brasileira. B. Técn. Petrobras, 1(2):225-233.

Roth P.H. 1973. Calcareous nannofossils - Leg 17, Deep Sea Drilling Project. Initial Reports of the DSDP, 17:695-795.

Roth P.H. 1978. Cretaceous nannoplankton biostratigraphy and oceanography of the northwestern Atlantic Ocean. Initial Reports of the DSDP, 44:731-760.
Siesser, W.G. 1994. Historical background of coccolithophore studies. In: Winter, A., Siesser, W.G. eds. 1994. Coccolithophores. Cambridge: Cambridge Univ. Press, p. 1-11.

Sissingh W. 1977. Biostratigraphy of Cretaceous calcareous nannoplankton. Geologie en Mijnbouw, 56: 37-65.

Sorby H.C. 1861. On the organic origin of the socalled "crystalloids" of the chalk. Annals and Magazine of Natural History, serie 3, 8:193-200.

Stradner H. 1963. New contributions to Mesozoic stratigraphy by means of nannofossils. In: WORLD PETROLEUM CONGRESS, 6. Proc... Section 1, Paper 4: 167-183.

Thierstein H.R. 1976. Mesozoic calcareous nannoplankton biostratigraphy of marine sediments. Marine Micropaleontology, 1:325-362.

Tizard T.H., Moseley H.N., Buchahan J.V., Murray J. 1885. Narrative of the Cruise of HMS Challenger. In: Report of the Scientific Results of the Voyage of the HMS Challenger during the years 1873-1876. Edinburg, 1:511-1110.

Troelsen J.C., Quadros L.P. 1971. Distribuição bioestratigráfica dos nanofósseis em sedimentos marinhos (Aptiano-Mioceno) do Brasil. An. Acad. brasil. Geoc., 43(suplemento):577-609.

Wallich G.C. 1861. Remarks on some novel phases of organic life, and on the boring powers of minute annelids, at great depths in the sea. Annals and Magazine of Natural History, Series 3, 8:52-58.

Winchester S. 2004. O mapa que mudou o mundo. Rio de Janeiro: Editora Record. 412 p.

Zerfass G.S.A., Andrade E.J. 2008. Foraminíferos e Bioestratigrafia: uma abordagem didática. Terre Didatica, 3(1):18-35. 